\title{
How Electrons Spin
}

\author{
Charles T. Sebens \\ University of California, San Diego
}

May 26, 2018

arXiv v.1

\begin{abstract}
There are a number of reasons to think that the electron cannot truly be spinning. Given how small the electron is generally taken to be, it would have to rotate superluminally to have the right angular momentum and magnetic moment. Also, the electron's gyromagnetic ratio is twice the value one would expect for an ordinary classical rotating charged body. These obstacles can be overcome by examining the flow of mass and charge in the Dirac field (interpreted as giving the classical state of the electron). Superluminal velocities are avoided because the electron's mass and charge are spread over sufficiently large distances that neither the velocity of mass flow nor the velocity of charge flow need to exceed the speed of light. The electron's gyromagnetic ratio is twice the expected value because its charge rotates twice as fast as its mass.
\end{abstract}

\section{Contents}

1 Introduction 2

2 The Obstacles 4

3 The Electromagnetic Field $\quad 6$

4 The Dirac Field $\quad 9$

5 The Dirac Sea 16

6 Conclusion $\quad 20$ 


\section{Introduction}

In quantum theories, we speak of electrons as having "spin." The reason we use this term is that electrons possess an angular momentum and a magnetic moment, just as one would expect for a rotating charged body. However, textbooks frequently warn students against thinking of the electron as actually rotating, or even being in some quantum superposition of different rotating motions. There are three serious obstacles to regarding the electron as a spinning object:

1. Given certain upper limits on the size of an electron, the electron's mass would have to rotate faster than the speed of light in order for the electron to have the correct angular momentum.

2. Similarly, the electron's charge would have to rotate faster than the speed of light in order to generate the correct magnetic moment.

3. A simple classical calculation of the electron's gyromagnetic ratio yields the wrong answer - off by a factor of (approximately) 2 .

These obstacles can be overcome by taking the electron's classical state (the state which enters superpositions) to be a state of the Dirac field. The Dirac field possesses mass and charge. One can define velocities describing the flow of mass and the flow of charge. The first two obstacles are addressed by the fact that the electron's mass and charge are spread over sufficiently large distances that neither the velocity of mass flow nor the velocity of charge flow need to exceed the speed of light. The electron's gyromagnetic ratio is twice the expected value because its charge rotates twice as fast as its mass.

In the next section I present the three obstacles above in more detail. Then, I consider how the obstacles are modified by the fact that some of the electron's mass is in the electromagnetic field that surrounds it. The mass in the electromagnetic field rotates around the electron and thus contributes to its angular momentum. Because the amount of mass in the electromagnetic field ultimately turns out to be small, this is not the dominant contribution to the electron's angular momentum. But, the idea of mass rotating in a classical field appears again when we consider the Dirac field which describes the electron itself. After an initial examination of this flow of mass and charge in the Dirac field, the above obstacles are addressed using a classical field implementation of the Dirac sea (which does not involve positing an infinite sea of electrons). The electron has a minimum size which is sufficiently large that the worries about superluminal rotation raised above are avoided. The factor of two in the gyromagnetic ratio of the electron is explained by examining the differing speeds at which mass and charge rotate in the Dirac field.

Before jumping into all of that, let me explain the focus on classical field theory in a paper about electron spin (a supposedly quantum phenomenon). When one moves from classical field theory to a quantum description of the electron within the quantum field theory of quantum electrodynamics, the classical Dirac and electromagnetic fields 
are quantized. Instead of representing the electron by a definite state of the Dirac field generating a definite electromagnetic field, we represent the electron by a superposition of different field states - a wave functional that assigns amplitudes to different possible classical states of the fields. The dynamics of this quantum state are determined by a wave functional version of the Schrödinger equation and can be calculated using path integrals which sum contributions from different possible evolutions of the fields (different possible paths through the space of field configurations). Seeing that the three obstacles above can be surmounted for each classical state of the two fields makes the nature of spin in this quantum theory of those fields much less mysterious. The electron simply enters superpositions of different states of rotation.

In the previous paragraph I assumed a perspective on quantum field theory that starts from a classical interpretation of the Dirac field, treating it as a relativistic classical field. Carroll (2016) gives a nice explanation of this classical interpretation of the Dirac equation:

"What about the Klein-Gordon and Dirac equations? These were, indeed, originally developed as 'relativistic versions of the non-relativistic Schrödinger equation,' but that's not what they ended up being useful for. ... The Klein-Gordon and Dirac equations are actually not quantum at all - they are classical field equations, just like Maxwell's equations are for electromagnetism and Einstein's equation is for the metric tensor of gravity. They aren't usually taught that way, in part because (unlike E\&M and gravity) there aren't any macroscopic classical fields in Nature that obey those equations." 1

Carroll then goes on to explain that the quantum state can be represented as a wave functional obeying a version of the Schrödinger equation.

There is an alternative perspective on quantum field theory which begins instead from a quantum interpretation of the Dirac field, treating it as a relativistic quantum wave function for the electron. On this quantum interpretation, the electron's mass and charge are not spread out. The electron is treated as a point particle in a superposition of different locations. From this relativistic quantum particle theory, one moves to a full quantum field theory by a process that is sometimes (inaptly) termed "second quantization." I think this transition is best understood as simply moving from a relativistic quantum theory of a single particle to a theory with a variable number of particles. Instead of having a wave function which assigns amplitudes to possible spatial locations for a single particle, one uses a wave function which assigns amplitudes

\footnotetext{
${ }^{1}$ Carroll's point at the end of the quote is important (see also Duncan, 2012, chapter 8). The motivation for studying the classical Dirac field in this paper is not that classical Dirac field theory emerges as an approximate description at the macroscopic level of what is happening microscopically according to the full quantum field theory. The motivation is that quantum field theory describes superpositions of states of the classical Dirac field. Classical Dirac field theory plays a key role in the foundations of quantum electrodynamics.
} 
to possible spatial arrangements of any number of particles (to points in the disjoint union of all $N$-particle configuration spaces).

These two perspectives are often seen as different ways of formulating the very same physical theory. ${ }^{2}$ However, in trying to understand what really exists in nature it is tempting to ask which perspective better reflects reality. Put another way: Is quantum field theory fundamentally a theory of fields or particles? This is a tough question and I will not attempt to settle it here (or even to develop the alternatives in much detail). I seek only to display a single virtue of the first perspective: it allows us to understand electrons as truly spinning. Adopting the first perspective is compatible with a number of different strategies for interpreting quantum field theory as it leaves open many foundational questions, such as: Does the wave functional ever collapse? Is there any additional ontology beyond the wave functional? Are there many worlds or is there just one?

What follows is a project of interpretation, not modification. It is generally agreed that the equations of our best physical theories describe an electron which has a property called "spin" but does not actually rotate. Here I present an alternative interpretation of the very same equations. There is no need to modify these equations so that they describe a rotating electron. Interpreted properly, they already do.

\section{The Obstacles}

The first obstacle to regarding the electron as truly spinning is that it must rotate superluminally in order to have the correct angular momentum. One estimate for the radius of the electron (which will be explained shortly) is the classical electron radius, $\frac{e^{2}}{m c^{2}} \approx 10^{-13} \mathrm{~cm}$. If you assume that the angular momentum of the electron is due entirely to the spinning of a sphere with this radius and the mass of the electron, points on the edge of the sphere would have to be moving superluminally (Griffiths, 2005, problem 4.25). To get an angular momentum of $\frac{\hbar}{2}$ with subluminal rotation speeds, the electron's radius must be greater than (roughly) the Compton radius of the electron, $\frac{\hbar}{m c} \approx 10^{-11} \mathrm{~cm}$. The relation between velocity at the equator $v$ and angular momentum

\footnotetext{
${ }^{2}$ For more on the first perspective, see Hatfield (1992, chapters 10 and 11); Valentini (1992, chapter 4); Valentini (1996); Peskin \& Schroeder (1995, chapter 3); Weinberg (1999, pg. 241-242); Tong (2006, chapters 2 and 4); Struyve (2010); Wallace (forthcoming). For more on the second, see Schweber (1961, chapters 6-8); Teller (1995, chapter 3); Colin (2004, section 3); Dürr et al. (2005, section 3); Wallace (forthcoming). There are reasons why it is more difficult to take the first perspective regarding fermions (like the electron) than it is for bosons (like the photon); see the above references for the first perspective, many of which employ Grassman numbers in quantizing fermionic fields. In response, one might take the second perspective regarding fermionic fields and the first for bosonic fields, treating fermions as fundamentally particle-like and bosons as fundamentally field-like (e.g., see Bohm \& Hiley, 1993). Instead, the project I pursue here proceeds under the assumption that it is possible to adopt a unified perspective that treats both fermions and bosons as fundamentally field-like. By taking on such an assumption, I am betting that the challenges facing the first perspective can be resolved in a satisfactory way.
} 
$|\vec{L}|$ for a spherical shell of mass $m$ and radius $R$ is

$$
|\vec{L}|=\frac{2}{3} m v R
$$

Setting $|\vec{L}|=\frac{\hbar}{2}$ and $v=c$ then solving for $R$ yields a radius on the order of the Compton radius,

$$
R=\frac{4}{3} \frac{\hbar}{m c} .
$$

Rejecting this picture of a spinning extended electron, one might imagine the mass of the electron to be confined to a single point. ${ }^{3}$ If this were so, the electron's angular momentum - as calculated from the usual definition of angular momentum in terms of the linear momentum and displacement from the body's center of a body's parts - would be zero (as none of the point electron's mass is displaced from its center). One might respond that in quantum physics we are forced to revise this "classical" definition of angular momentum and allow point particles to posses angular momentum. The following sections show that there is no need to so radically revise our understanding of angular momentum.

The second obstacle is that an electron with the classical electron radius would have to spin superluminally to produce the correct magnetic moment. Assuming the magnetic moment is generated by a spinning sphere of charge imposes essentially the same minimum radius for the electron as the first obstacle - the Compton radius. The relation between velocity and magnetic moment $|\vec{m}|$ for a spherical shell of charge $-e$ is

$$
|\vec{m}|=\frac{e R v}{3 c}
$$

(Rohrlich, 2007, pg. 127). Inserting $v=c$ and $|\vec{m}|=\frac{e \hbar}{2 m c}$ (the Bohr magneton) yields a radius of

$$
R=\frac{3}{2} \frac{\hbar}{m c} .
$$

The third obstacle to regarding the electron as spinning is that its gyromagnetic ratio (the ratio of magnetic moment to angular momentum) differs from the simplest classical estimate (Griffiths, 1999, problem 5.56; Jackson, 1999, pg. 187):

$$
\frac{|\vec{m}|}{|\vec{L}|}=\frac{e}{2 m c}
$$

I stress that this is the simplest estimate and not the classical gyromagnetic ratio because its derivation requires two important assumptions beyond axial symmetry, each of which will be called into question later: (1) the mass $m$ and charge $-e$ are both distributed in the same way, i.e., mass density is proportional to charge density, and (2) the mass and charge rotate at the same rate. With these assumptions in place, the derived $6)$

${ }^{3}$ The fact that there is mass in the electromagnetic field makes this difficult to imagine (see footnote 
gyromagnetic ratio is independent of the rate of rotation and the distribution of mass and charge. The actual gyromagnetic ratio of the electron is twice this estimate,

$$
\frac{|\vec{m}|}{|\vec{L}|}=\frac{e}{m c}
$$

as its angular momentum is $\frac{\hbar}{2}$ and its magnetic moment is the Bohr magneton, $\frac{e \hbar}{2 m c}$ (ignoring the anomalous magnetic moment).

The physicists who first proposed the idea of electron spin were aware of these obstacles. Ralph Kronig was the first to propose a spinning electron to explain the fine structure of atomic line spectra (in 1925), but he did not publish his results because there were too many problems with his idea. One of these problems was that the electron would have to rotate superluminally (Tomonaga, 1997, pg. 35). Independently of Kronig, George Uhlenbeck and Samuel Goudsmit had the same idea. Uhlenbeck spoke with Hendrik Lorentz about the proposal and Lorentz brought up the problem of superluminal rotation (among others). After speaking with Lorentz, Uhlenbeck no longer wanted to publish. But, it was too late. His advisor, Paul Ehrenfest, had already sent the paper off. Uhlenbeck recalls Ehrenfest attempting to reassure the pair by saying: "You are both young enough to be able to afford a stupidity!" (Uhlenbeck, 1976, pg. 47; see also Goudsmit, 1998). Uhlenbeck and Goudsmit were also aware of the gyromagnetic ratio problem, but they were not so troubled by it. They understood that the classical calculation of the gyromagnetic ratio has assumptions which can be denied (e.g., if the electron's mass is spread evenly throughout a sphere but its charge is confined to the sphere's surface; Uhlenbeck, 1976, pg. 47; Pais, 1989, pg. 39).

\section{The Electromagnetic Field}

Before going on to model the electron using the Dirac field, it is worthwhile to consider how the above obstacles are altered by the taking the mass of the electromagnetic field into account. The electromagnetic field possesses energy and associated with that energy, by the relativistic equivalence of mass and energy, is a relativistic mass (Einstein, 1906; Lange, 2002; Sebens, forthcoming). In Gaussian units, the density of energy is

$$
\rho_{f}^{\mathcal{E}}=\frac{1}{8 \pi}\left(|\vec{E}|^{2}+|\vec{B}|^{2}\right)
$$

and the density of relativistic mass is

$$
\rho_{f}=\frac{\rho_{f}^{\mathcal{E}}}{c^{2}}=\frac{1}{8 \pi c^{2}}\left(|\vec{E}|^{2}+|\vec{B}|^{2}\right) .
$$

The $f$ subscript indicates that these are properties of the electromagnetic field. The total mass of the electron is the sum of this electromagnetic mass plus any mass possessed by 
the electron itself. ${ }^{4}$ The mass of the electromagnetic field moves with a certain velocity that can be expressed in terms of the Poynting vector, $\vec{S}=\frac{c}{4 \pi} \vec{E} \times \vec{B}$, which gives the energy flux density of the field. The field velocity ${ }^{5}$ can be found by dividing the energy flux density $\vec{S}$ by the energy density $\rho_{f}^{\mathcal{E}}$ or, equivalently, by dividing the momentum density of the field,

$$
\vec{G}_{f}=\frac{\vec{S}}{c^{2}}=\frac{1}{4 \pi c} \vec{E} \times \vec{B}
$$

by its mass density (8),

$$
\vec{v}_{f}=\frac{\vec{G}_{f}}{\rho_{f}}=\frac{\vec{S}}{\rho_{f}^{\mathcal{E}}} .
$$

Looking at the field lines around a charged magnetic dipole, such as an electron, it is clear from (9) and (10) that the field mass circles the axis picked out by the dipole, as depicted in figure 1 (Feynman et al. , 1964, chapter 27; Lange, 2002, chapter 8).

The fact that some (or perhaps all) of the mass of the electron is located outside the bounds of the electron itself $^{6}$ and rotating is helpful for addressing the first obstacle - getting a large angular momentum without moving superluminally is easier if the mass is more spread out. Also, there is no danger of the mass in the electromagnetic field moving superluminally since the magnitude of the field velocity in (10) is maximized at $c$ when $\vec{E}$ is perpendicular to $\vec{B}$ and $|\vec{E}|=|\vec{B}|$.

We are now in a position to see where the classical electron radius comes from and to see why it is a completely unreasonable estimate to use in motivating the first obstacle. Let's work up to that slowly. First, note that the smaller the electron is, the greater the mass in the electric and magnetic fields surrounding the electron. Keeping the total mass of the electron fixed, the smaller the electron is, the less mass it itself possess. If we imagine making the electron as small as possible, ${ }^{7}$ putting all of its mass in the electromagnetic field, we arrive at a radius for the electron that we can call the "electromagnetic radius," on the order of ${ }^{8} 10^{-12} \mathrm{~cm}$ (Uhlenbeck, 1976, pg. 47; Pais, 1989, pg. 39; MacGregor, 1992, chapter 8). The classical electron radius was arrived at through similar reasoning applied before the electron's magnetic moment was discovered. It was assumed that the electron's mass comes entirely from its electric field. If we take the electron's charge to be distributed evenly over a spherical shell, the radius calculated

"I will use the phrase "the electron itself" to refer to the bare electron, distinct from the electromagnetic field that surrounds it. This is to be contrasted with the dressed electron, which includes both the electron itself and its electromagnetic field.

${ }^{5}$ This field velocity appears in Poincaré (1900); Holland (1993, section 12.6.2); Lange (2002, box 8.3); Sebens (forthcoming).

${ }^{6}$ Sometimes you see it said that a portion of the electron's mass is electromagnetic in origin, which seems to suggest that although this portion of mass originates in the energy of the electromagnetic field it is possessed by and located at the electron itself. I have argued against such an understanding of electromagnetic mass in Sebens (forthcoming). The electromagnetic mass is located in the electromagnetic field.

${ }^{7}$ If we were willing to make the mass of the electron itself negative, its radius could be even smaller (Pearle, 1982, pg. 214).

${ }^{8}$ The exact number depends on the way the electron's charge is distributed and how that charge flows. 


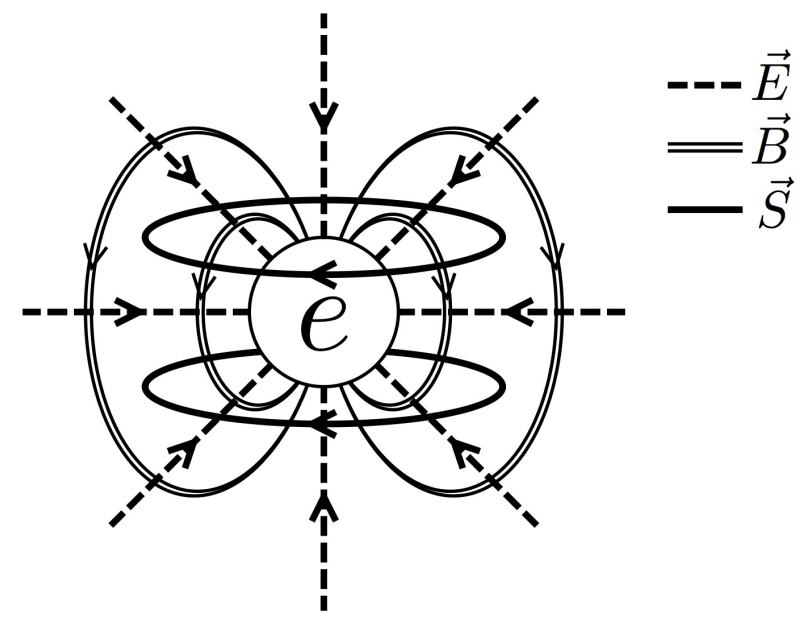

Figure 1: This figure depicts the electric and magnetic fields produced by the electron's charge and magnetic dipole moment. Also shown is the Poynting vector $\vec{S}$ which indicates that the mass of the electromagnetic field rotates about the axis picked out by the electron's magnetic moment.

in this way would be

$$
R=\frac{e^{2}}{2 m c^{2}},
$$

as the energy in the electric field is $\frac{e^{2}}{2 R}$. Ignoring the prefactor (which is dependent on the way the charge is distributed), we get the classical electron radius (Feynman et al. , 1964, section 38-3, Rohrlich, 2007, section 6-1),

$$
R=\frac{e^{2}}{m c^{2}} \approx 2.82 \times 10^{-13} \mathrm{~cm}
$$

an order of magnitude smaller than the electromagnetic radius as the amount of energy in the magnetic field is much greater than the amount in the electric field. Neither of these radii should prompt worries of superluminal mass flow. If the mass of the electron resides entirely in its electromagnetic field then the electron itself is massless and energyless. It doesn't matter how fast it's spinning since it itself won't have any angular momentum. The angular momentum is entirely in the field and the mass of the field cannot move superluminally.

Understanding that the electromagnetic field possesses mass does little to alter the second obstacle. Although the electron's mass bleeds into the field, its charge does not.

The third obstacle is complicated by the existence of field mass. The simple calculation of the gyromagnetic ratio for a spinning charged body given above (5) was the ratio of the magnetic moment produced by a spinning body to the angular momentum of that body itself. But, once we recognize that some of the mass we associate with that body is actually in its electromagnetic field, we must take the field's angular momentum into consideration when calculating the gyromagnetic ratio. Here is one illustrative way 
of doing so. The electric and magnetic fields for a charged magnetic dipole are

$$
\begin{aligned}
\vec{E} & =-e \frac{\vec{x}}{|\vec{x}|^{3}} \\
\vec{B} & =\frac{3(\vec{m} \cdot \vec{x}) \vec{x}}{|\vec{x}|^{5}}-\frac{\vec{m}}{|\vec{x}|^{3}} .
\end{aligned}
$$

If we assume that the charge is uniformly distributed over a spherical shell of radius $R$, so that the above electric field is only present outside this radius, and also that the only contribution to the angular momentum of the charged dipole is the angular momentum in the fields outside this radius (because the entirety of the electron's mass resides in its electromagnetic field), we arrive at a gyromagnetic ratio of

$$
\frac{|\vec{m}|}{|\vec{L}|}=\frac{3 R c}{2 e}
$$

Unlike the simple calculation of the gyromagnetic ratio of an axially symmetric spinning charged body mentioned above (5), this result is radius dependent. ${ }^{9}$

We must input a radius for the electron if we are to compare (3) to (5) and (6). One option would be to use the classical electron radius (12). However, we must be careful because the prefactors that were ignored in (12) are important in . In the earlier derivation of the angular momentum of the field, it was assumed that the charge was distributed uniformly over the surface of the sphere as in (11). Continuing with that assumption and plugging (11) into (3) yields a gyromagnetic ratio of

$$
\frac{|\vec{m}|}{|\vec{L}|}=\frac{3 e}{4 m c}
$$

closer to (6) than (5), but still incorrect. The classical electron radius was calculated by ignoring the magnetic field of the electron. Taking the magnetic field into consideration and using the electromagnetic radius instead of the classical radius would yield a gyromagnetic ratio which is much too large. So, the assumption that the mass of the electron is entirely in the electromagnetic field leads to trouble. Fortunately, it's not true. In section 5 we'll see that the electron is large enough that the mass of the electromagnetic field surrounding the electron is only a small fraction of the electron's total mass.

\section{The Dirac Field}

In the previous section we examined the flow of mass in the electromagnetic field surrounding the electron. In this section we ignore the electromagnetic field and focus exclusively on the flow of mass and charge of the electron itself (assuming, contra the

\footnotetext{
${ }^{9}$ I have only rarely seen the angular momentum of the electromagnetic field taken into account when calculating the gyromagnetic ratio of the electron (e.g., Corben, 1961; Giulini, 2008).
} 
previous section, that little of the electron's mass is in the electromagnetic field). We can understand this flow of mass and charge by using the Dirac field to represent the state of the electron. In this section I make heavy use of the excellent account of spin given by Ohanian (1986). ${ }^{10}$

As was discussed in the introduction, the Dirac equation,

$$
i \hbar \frac{\partial \psi}{\partial t}=\left(\frac{\hbar c}{i} \gamma^{0} \vec{\gamma} \cdot \vec{\nabla}+m \gamma^{0} c^{2}\right) \psi
$$

can either be viewed as part of a relativistic quantum theory in which $\psi$ is a wave function (the quantum interpretation), or, as part of a relativistic field theory in which $\psi$ is a classical field (the classical interpretation). ${ }^{11}$ Here I adopt the second perspective and take $\psi$ to be a classical field. The classical Dirac field can be quantized, along with the classical electromagnetic field, to arrive at the quantum field theory of quantum electrodynamics. In the context of quantum electrodynamics, the electron is described by a superposition of different states for the classical Dirac field (a wave functional). In this paper, we will examine the classical field states which compose this superposition and see that our obstacles can be overcome for each such classical state. We will not need to go as far as quantizing the Dirac field. In none of the equations that appear here is $\psi$ an operator. At this level of physics, there are just two interacting classical fields - the Dirac field and the electromagnetic field. ${ }^{12}$

Much like the electromagnetic field, the Dirac field carries energy and momentum. Ignoring any interaction with the electromagnetic field, the energy and momentum

\footnotetext{
${ }^{10}$ Ohanian's analysis of spin differs from the one presented here in a number of important respects. Ohanian calls $\psi$ a "wave field" and treats it as a quantum wave function, not a classical field. He does not directly address the three obstacles raised in section 2 and does not make the moves in section 5 which are necessary to surmount them. He discusses the flow of energy and charge, but introduces neither a velocity of mass flow (22) nor a velocity of charge flow (25).

${ }^{11}$ As the Dirac field is sometimes interpreted as a wave function and sometimes as a classical field, one might naturally wonder if it is possible to interpret the electromagnetic field as a wave function instead of a classical field. Bialynicki-Birula (1996) defends the utility of such an interpretation (see also Good, 1957; Mignani et al. , 1974).

${ }^{12}$ Weyl (1932, pg. 216-217) explicitly considers and rejects the idea that the Dirac field should be treated as a classical field along the lines proposed here, comparing the idea to Schrödinger's original pre-Born-rule interpretation of his eponymous equation where the amplitude-squared of the wave function is interpreted as a charge density. It is true that before quantization the classical Dirac field does not provide an adequate theory of the electron (though such a theory works better than you might expect; see Crisp \& Jaynes, 1969; Jaynes, 1973; Barut, 1988; Barut \& Dowling, 1990). What matters for our purposes here is not the adequacy of classical Dirac field theory itself, but just the fact that it is this classical field theory which gets quantized to arrive at our best theory of the electron, quantum electrodynamics. (It is worth noting that Weyl, 1932 later treats the Dirac field like a classical field when quantizing it; see Pashby, 2012, pg. 451.)
} 
densities are given by: ${ }^{13,14}$

$$
\begin{aligned}
\rho_{d}^{\mathcal{E}} & =\frac{i \hbar}{2}\left(\psi^{\dagger} \frac{\partial \psi}{\partial t}-\frac{\partial \psi^{\dagger}}{\partial t} \psi\right) \\
& =m c^{2} \psi^{\dagger} \gamma^{0} \psi+\frac{\hbar c}{2 i}\left[\psi^{\dagger} \gamma^{0} \vec{\gamma} \cdot \vec{\nabla} \psi-\left(\vec{\nabla} \psi^{\dagger}\right) \cdot \gamma^{0} \vec{\gamma} \psi\right] \\
\vec{G}_{d} & =\frac{\hbar}{2 i}\left[\psi^{\dagger} \vec{\nabla} \psi-\left(\vec{\nabla} \psi^{\dagger}\right) \psi\right]+\frac{\hbar}{4} \vec{\nabla} \times\left(\psi^{\dagger} \vec{\sigma} \psi\right) .
\end{aligned}
$$

The $d$ subscript indicates that these are properties of the Dirac field. The second term in the momentum density gives the contribution from spin (Wentzel, 1949, pg. 181-182, Pauli, 1980, pg. 168, Ohanian, 1986, pg. 503). Because the spin contribution is a curl, it will not contribute to the total linear momentum of the electron. When the momentum density in (19) is used to calculate the angular momentum of an electron, the first term yields the orbital angular momentum and the second yields the spin angular momentum. The density of spin angular momentum derived from the second term in (19) is

$$
\frac{\hbar}{2} \psi^{\dagger} \vec{\sigma} \psi
$$

As we are here concerned with understanding spin, we will focus on states where the electron is at rest and the first term in (19) is everywhere zero.

Although I have not seen it done before, we can introduce a relativistic mass density and a velocity that describes the flow of mass (or energy) in just the same way as was done for the electromagnetic field in the previous section,

$$
\begin{aligned}
& \rho_{d}=\frac{\rho_{d}^{\mathcal{E}}}{c^{2}}=m \psi^{\dagger} \gamma^{0} \psi+\frac{\hbar}{2 i c}\left[\psi^{\dagger} \gamma^{0} \vec{\gamma} \cdot \vec{\nabla} \psi-\left(\vec{\nabla} \psi^{\dagger}\right) \cdot \gamma^{0} \vec{\gamma} \psi\right] \\
& \vec{v}_{d}=\frac{\vec{G}_{d}}{\rho_{d}}
\end{aligned}
$$

In contrast with the electromagnetic field, the Dirac field's energy density can be negative and thus its mass density can be negative as well.

In addition to the mass density and its flow, we can examine the charge density of the Dirac field and the flow of charge. The charge density and charge current density are

$$
\begin{aligned}
\rho_{d}^{q} & =-e \psi^{\dagger} \psi \\
\vec{J}_{d} & =-e c \psi^{\dagger} \gamma^{0} \vec{\gamma} \psi .
\end{aligned}
$$

\footnotetext{
${ }^{13}$ These two densities are components of the symmetrized stress-energy tensor for the Dirac field (Wentzel, 1949, section 20; Heitler, 1954, appendix 7; Weyl, 1932, pg. 218-221).

${ }^{14}$ Here $\gamma^{0}, \vec{\gamma}$, and $\vec{\sigma}$ are four-dimensional matrices, related to the two-dimensional Pauli spin matrices $\vec{\sigma}_{p}$ by

$$
\gamma^{0}=\left(\begin{array}{cc}
I & 0 \\
0 & -I
\end{array}\right) \quad \vec{\gamma}=\left(\begin{array}{cc}
0 & \vec{\sigma}_{p} \\
-\vec{\sigma}_{p} & 0
\end{array}\right) \quad \vec{\sigma}=\left(\begin{array}{cc}
\vec{\sigma}_{p} & 0 \\
0 & \vec{\sigma}_{p}
\end{array}\right)
$$


These densities act as source terms for Maxwell's equations. From the charge and current densities, we can define the velocity of charge flow as

$$
\vec{v}_{d}^{q}=\frac{\vec{J}_{d}}{\rho_{d}^{q}}=\frac{c \psi^{\dagger} \gamma^{0} \vec{\gamma} \psi}{\psi^{\dagger} \psi}
$$

From this definition, it follows that the charge velocity cannot exceed the speed of light (Bohm \& Hiley, 1993, section 10.4; Holland, 1993, section 12.2). Because of this light-speed limit, our second obstacle is automatically averted. Superluminal charge flow is impossible.

I should note that when a velocity for the Dirac field is discussed, it is usually steeped in the quantum interpretation of the Dirac field as a wave function (e.g., Dirac, 1958, section 69; Bjorken \& Drell, 1964, pg. 11; Heitler, 1954, pg. 106; Frenkel, 1934, sections 31 and 32 ). There exists a "velocity operator" $c \gamma^{0} \vec{\gamma}$ (or, equivalently, $c \vec{\alpha}$ ). ${ }^{15}$ The local expectation value of this operator, $c \psi^{\dagger} \gamma^{0} \vec{\gamma} \psi$, is taken to be the probability current density. The quantity $\psi^{\dagger} \psi$ is interpreted as a probability density. Dividing the probability current density by the probability density gives a Bohmian particle velocity, $\frac{c \psi^{\dagger} \gamma^{0} \vec{\gamma} \psi}{\psi^{\dagger} \psi}$, equal to the velocity of charge flow given above (25) and thus also capped at $c$ (Bohm, 1953, Bohm \& Hiley, 1993, section 10.4; de Broglie, 1960, pg. 203; Holland, 1993, equation 12.2.10). ${ }^{16}$ Because I am treating the Dirac field as a classical field, all of this quantum talk about the flow of probability is inappropriate. The classical Dirac field has a mass density and a charge density, but no probability density.

The reason the gyromagnetic ratio of the electron differs from the simple classical estimate (5) by a factor of two can be explained straightforwardly in the context of Dirac field theory using the velocities introduced above. ${ }^{17}$ In the simple estimate, we assumed that the mass and charge were rotating together at the same rate. Actually, the charge of the electron rotates twice as quickly as the mass. ${ }^{18}$ So, the magnetic moment is twice as large as you'd expect given the angular momentum.

This factor of two between the mass and charge velocity is a general feature of wave functions that describe an electron at rest. But, to see how it arises it will be helpful to

\footnotetext{
${ }^{15}$ Taking this to be the only notion of velocity for the Dirac field leads to an oddity: momentum and velocity are not parallel (Frenkel, 1934, pg. 329; Bjorken \& Drell, 1964, pg. 37). This oddity is remedied by using the velocity of mass flow introduced above (22).

${ }^{16}$ Taking the electron to be a point particle moving with this velocity leads to the possibility of understanding the electron's angular momentum and magnetic moment as generated by the electron's motion within its wave function. Bohm \& Hiley (1993, pg. 218) argue that: "in the Dirac theory, the magnetic moment usually attributed to the 'spin' can actually be attributed to a circulating movement of a point particle, and not that of an extended spinning object."

${ }^{17}$ It is generally agreed that there exists some explanation of the gyromagnetic ratio in the context of the Dirac equation. The task here is to better understand what sort of explanation is available (compare Ohanian, 1986, pg. 504 and Bjorken \& Drell, 1964, section 1.4).

${ }^{18}$ How could charge move at a different velocity than mass? Imagine you're describing a fluid flowing through pipes using certain mass and charge densities. On closer inspection, the fluid turns out to be made of two kinds of particles - heavy neutral particles and light positively charged particles. Sometimes the charged particles flow faster than the neutral ones and the velocity of charge flow is greater than the velocity of mass flow. Sometimes the heavy particles flow faster than the light ones and the velocity of mass flow is greater than the velocity of charge flow.
} 
start with a particular illustrative example wave function. Here is a simple instantaneous state of the Dirac field which we can use as a first approximation towards representing a single electron which is (at this moment) at rest with $z$-spin up (Ohanian, 1986, equation 14; Bjorken \& Drell, 1964, equation 3.32)

$$
\psi=\left(\frac{1}{\pi d^{2}}\right)^{3 / 4} e^{-|\vec{x}|^{2} / 2 d^{2}}\left(\begin{array}{l}
1 \\
0 \\
0 \\
0
\end{array}\right)
$$

The mass and charge are both localized in a Gaussian wave packet of width $d$. The reason for calling this a single electron state is that the integral of the charge density over all of space is $-e$.

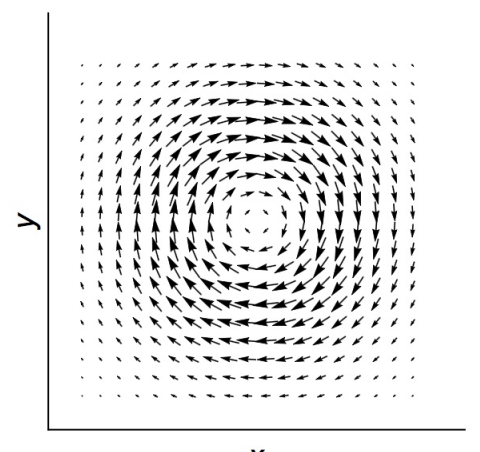

$x$

Momentum Density

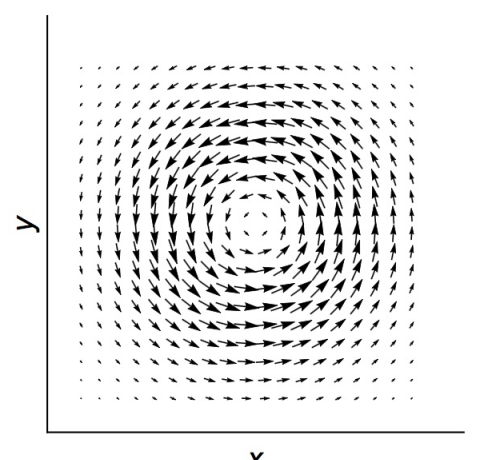

Current Density

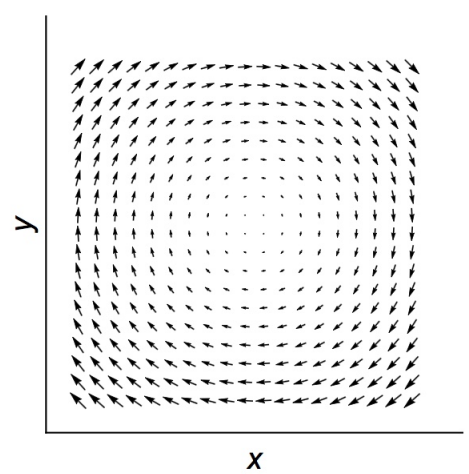

Mass Velocity

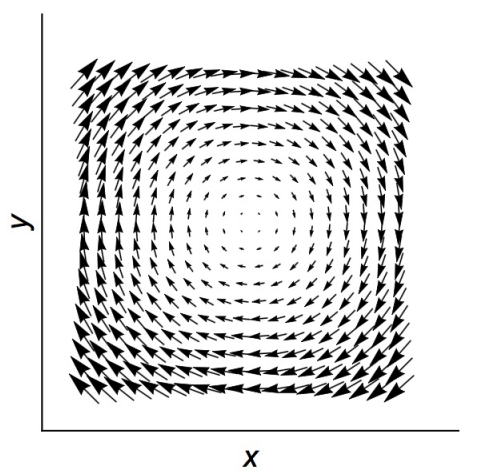

Charge Velocity

Figure 2: These plots depict the flow of mass and charge for the state of an electron at rest given in (26). The first two plots give the momentum density (27) and mass velocity (28). The second two plots give the magnetization current density (31) and the corresponding contribution to the charge velocity (32) (for the corrected state (36), these plots give the total charge current density and charge velocity). The two velocity plots use the same scale to highlight that the charge velocity is twice the mass velocity. 
The momentum density for this state is

$$
\vec{G}_{d}=\frac{\hbar}{2}\left(\frac{1}{\pi d^{2}}\right)^{3 / 2} e^{-|\vec{x}|^{2} / d^{2}} \frac{\vec{x} \times \hat{z}}{d^{2}}
$$

calculated via (19) where only the second term is non-zero. From this expression, it is clear that mass and energy are flowing around the $z$-axis (see figure 2). The mass velocity for this state can be calculated by dividing this momentum density by the mass density as in (22),

$$
\vec{v}_{d}=\frac{\hbar}{2 m} \frac{\vec{x} \times \hat{z}}{d^{2}}=\frac{\hbar r}{2 m d^{2}} \hat{\theta} .
$$

The second expression gives the velocity in cylindrical coordinates. This equation shows that the mass flows everywhere about the $z$-axis at constant angular velocity. The electron's mass appears ${ }^{19}$ to rotate like a solid object.

To calculate the velocity at which charge flows, it is useful to first expand the current density using the free Dirac equation as follows ${ }^{20}$

$$
-e c \psi^{\dagger} \gamma^{0} \vec{\gamma} \psi=\underbrace{\frac{i e \hbar}{2 m}\left\{\psi^{\dagger} \gamma^{0} \vec{\nabla} \psi-\left(\vec{\nabla} \psi^{\dagger}\right) \gamma^{0} \psi\right\}}_{(1)} \underbrace{-\frac{e \hbar}{2 m} \vec{\nabla} \times\left(\psi^{\dagger} \gamma^{0} \vec{\sigma} \psi\right)}_{(2)} \underbrace{+\frac{i e \hbar}{2 m c} \frac{\partial}{\partial t}\left(\psi^{\dagger} \vec{\gamma} \psi\right)}_{\text {(3) }}
$$

The three terms in the expansion are the convection current density, the magnetization current density, and the polarization current density. As was the case for the momentum density (19), the first term is zero for an electron at rest. The second two terms give the contribution to the charge current from spin. For the moment, let us focus on the magnetization current density. The magnetization current density in (29) corresponds to a magnetic moment density of

$$
-\frac{e \hbar}{2 m c} \psi^{\dagger} \gamma^{0} \vec{\sigma} \psi
$$

where the prefactor is the Bohr magneton. ${ }^{21}$ The ratio of the magnitude of this magnetic moment density to the magnitude of the angular moment density in (20) for the state in $(26)$ is $\frac{e}{m c}$, the correct gyromagnetic ratio for the electron (6). The magnetization current density,

$$
\frac{-e \hbar}{2 m c}\left(\frac{1}{\pi d^{2}}\right)^{3 / 2} e^{-|\vec{x}|^{2} / d^{2}} \frac{\vec{x} \times \hat{z}}{d^{2}},
$$

makes a contribution to the velocity of charge flow, calculated via (25), of

$$
\frac{\hbar}{m} \frac{\vec{x} \times \hat{z}}{d^{2}}=\frac{\hbar r}{m d^{2}} \hat{\theta}
$$

\footnotetext{
${ }^{19}$ I use the qualification "appears" because, as will be explained shortly, (26) is not an entirely satisfactory approximation to the state of an electron.

${ }^{20}$ This expansion appears in Frenkel (1934, pg. 321-322); Ohanian (1986, pg. 504).

${ }^{21}$ See Jackson (1999, section 5.6); Ohanian (1986, pg. 504).
} 
The contribution to the velocity of charge flow which determines the electron's magnetic moment (32) is twice the velocity of mass flow which determines the electron's angular momentum (28).

The factor of two between these velocities is not a peculiar feature of the chosen state, but will hold for any electron state in the non-relativistic limit. In general, the contribution to the moment density $\vec{G}_{d}$ from spin is $\frac{\hbar}{4} \vec{\nabla} \times\left(\psi^{\dagger} \vec{\sigma} \psi\right)$ - the second term in (19). In the non-relativistic limit, the relativistic mass density is approximately $m \psi^{\dagger} \gamma^{0} \psi$ - the first term in (21). Dividing these, as in (22), gives a contribution to the velocity of mass flow from spin of

$$
\frac{\hbar}{4 m} \frac{\vec{\nabla} \times\left(\psi^{\dagger} \vec{\sigma} \psi\right)}{\psi^{\dagger} \gamma^{0} \psi}
$$

The velocity associated with the electron's spin magnetic moment can be derived from the magnetization current density - the second term in (29). Dividing the magnetization current density by the charge density (23) as in (25) yields a contribution to the charge velocity of

$$
\frac{\hbar}{2 m} \frac{\vec{\nabla} \times\left(\psi^{\dagger} \gamma^{0} \vec{\sigma} \psi\right)}{\psi^{\dagger} \psi} .
$$

It is clear that (34) is twice (33) (up to factors of $\gamma^{0}$ which we will return to later).

Addressing our obstacles in the context of the Dirac equation has enabled significant progress, but there remain three serious shortcomings to the account given thus far which will be resolved in the following section. First, we have only been able to say (somewhat awkwardly) that a certain contribution to the velocity of charge flow is twice the velocity of mass flow and not that the actual velocity of charge flow is twice the velocity of mass flow. In fact, it is easy to see that the velocity of charge flow is zero for the state in (26) as the charge current density calculated from (24) is clearly zero. The first term in the current expansion (29) is also zero. Thus, one can see that the third term in (29) (the polarization current density) will exactly cancel the second (the magnetization current density). Because of this cancellation, no magnetic field is being produced by an electron in this state. If we are to account for the magnetic field around an electron at rest, the electron's charge must actually rotate.

Second, the velocities in (28) and (32) are unbounded, becoming superluminal as $r$ becomes very large. The fact that (32) becomes infinite is less troubling because, as was just discussed, it is cancelled by the contribution to the charge velocity from the polarization current. Also, as was mentioned earlier, it can be shown in general that the charge velocity cannot exceed $c$. The fact that (28) becomes superluminal is a real problem.

Third, there are problems which arise if the electron is too small and as of yet we have no reason to think it's large enough to avoid these problems. If the electron is too small, we face our first two obstacles concerning superluminal rotation. Also, if the electron is too small we will not be able to ignore the mass in the electromagnetic field 
when calculating the gyromagnetic ratio (as was done in this section but not the last). Looking at (26) it appears that the size of the electron is an entirely contingent matter depending on the state of the Dirac field. By decreasing $d$, the electron can be made arbitrarily small.

\section{The Dirac Sea}

The three problems raised at the end of the previous section can be resolved by restricting the allowed states of the Dirac field to those formed by superposing positive frequency modes. Such a restriction can be motivated as a way of implementing the idea of a Dirac sea in the context of classical Dirac field theory, though this move does not involve the postulation of an infinite sea of electrons.

In this section we will continue to restrict our attention to the free Dirac equation, putting aside issues of self-interaction - the electron is treated as blind to the electromagnetic field it generates - but still confronting issues of self-energy - the energies of the Dirac and electromagnetic fields are both taken into account in the following analysis.

The free Dirac equation admits of plane wave solutions with definite ${ }^{22}$ momentum $\vec{p}$ and time dependence given by either $e^{-i E t / \hbar}$ (positive frequency) or $e^{i E t / \hbar}$ (negative frequency), where $E$ is the energy associated with that momentum, $E=\sqrt{|\vec{p}|^{2} c^{2}+m^{2} c^{4}}$ (Bjorken \& Drell, 1964, chapter 3). From (18), it is clear that the positive frequency plane waves have uniform positive energy density and the negative frequency plane waves have uniform negative energy density.

On a quantum interpretation of the Dirac field, one would say that these plane wave solutions are eigenstates of momentum and energy where the energy eigenvalues may be positive or negative. The existence of such negative energy states proved both a blessing and a curse for early applications of the Dirac equation. To retain the blessing while dispelling the curse, Dirac proposed his hole theory according to which all of the negative energy states are filled (Dirac, 1930). By Pauli exclusion, any additional electrons must sit atop this "Dirac sea." The filled sea is taken to set the zero level for energy and charge. If any electron is excited out of the sea, the hole it leaves behind acts like a particle with equal mass and opposite charge to the electron - a positron. The possibility of creating such holes in the Dirac sea is essential for understanding many phenomena (including vacuum polarization, which would be important in a more thorough analysis of the Dirac and electromagnetic fields of an electron at rest). However, the discussion here will be simplified by assuming the Dirac sea to be filled. It is this rise in sea level, not the presence of holes, which is the key to solving our remaining problems.

Although the Dirac sea is generally explained in these quantum mechanical terms,

\footnotetext{
${ }^{22}$ On a classical interpretation of the Dirac field, by saying the momentum is "definite" I mean that the momentum density (19) is uniform.
} 
the simplified version of it described above - where the sea is always filled - can be easily implemented in the context of the classical interpretation of the Dirac equation where the Dirac field is viewed as a classical field. The change that must be made to classical Dirac field theory as presented in the previous section is not any change to the dynamical laws, but simply a shrinking of the space of possible states. Any state of the Dirac field which has a Fourier decomposition that includes negative frequency modes is forbidden. All allowed physical states are to be formed entirely from positive energy modes. Let us call this theory "restricted Dirac field theory." Restricted Dirac field theory incorporates important features of the Dirac sea without positing an infinite sea of electrons pervading all of space.

A different modification to classical Dirac field theory would be necessary to fully implement the idea of the Dirac sea (including the possibility of holes) and would certainly be required if we were considering interactions that could take states composed only of positive frequency modes into states containing both positive and negative frequency modes. This is a topic I hope to examine in future work. I expect that in such a theory both positive and negative frequency modes would be allowed, but the equation for charge density (23) would be revised so that negative frequency modes carry positive charge (as these modes would be associated with positrons). To keep things relatively simple, we will not consider such a full implementation of the Dirac sea here. We will focus on restricted Dirac field theory.

As was first examined by Weisskopf (1934a,b, 1939), the electromagnetic energy divergence - which arises because the amount of energy in an electron's electromagnetic field goes rapidly to infinity as its radius is decreased - is tamed in the context of hole theory (Schweber, 1994, section 2.5.3). Weisskopf's handling of this divergence in hole theory has been incorporated into the modern understanding of mass renormalization within quantum electrodynamics. ${ }^{23}$ The crucial insight from Weisskopf's analysis for our task at hand is expressed well by Heitler (1954, pg. 299). He writes that the taming of the electromagnetic self-energy divergence for an electron at rest "is a consequence of the hole theory and the Pauli [exclusion] principle":

"Consider an electron represented by a very small wave packet in coordinate space. In momentum space this would be represented by a distribution including negative energy states. The latter, however, are filled with vacuum electrons. Consequently, the negative energy contributions to the wave function must be eliminated and the electron cannot be a wave packet of infinitely small size but must have a finite extension (of the order $\frac{\hbar}{m c}$ [the Compton radius], as one easily finds). Consequently the static self-energy will be diminished also."

This explanation is given from within the quantum interpretation of the Dirac field as

\footnotetext{
${ }^{23}$ It is cited in relation to the Feynman diagram approach by Schweber (1961, pg. 513); Bjorken \& Drell (1964, pg. 165); Gottfried \& Weisskopf (1986, section II.D.2).
} 
wave function. But, it contains lessons that carry over to the classical interpretation: There is a limit on the minimum size wave packet that one can construct from the positive frequency modes of the Dirac field that are available in restricted Dirac field theory. The mass and charge of the electron cannot be confined to an arbitrarily small volume. $^{24}$ Because the charge of the electron is spread over such a large packet, the electromagnetic contribution to the energy (and mass) of an electron at rest is small and can be ignored when calculating the gyromagnetic ratio to a first approximation (as was done in the previous section). In section 2 we saw that in order to avoid the superluminal rotation speeds forced upon us in our first two obstacles, the electron must be at least as large as the Compton radius. The classical implementation of the Dirac sea delivers that minimum size.

Let us return to the instantaneous electron state in (26). In restricted Dirac field theory, this state is forbidden as it includes both positive and negative frequency modes. To find a similar state that is allowed, we can simply delete the negative frequency modes from the Fourier decomposition of (26). ${ }^{25}$ This yields ${ }^{26}$

$$
\psi=\frac{1}{2}\left(\frac{d^{2}}{\pi \hbar^{2}}\right)^{3 / 4}\left(\frac{1}{2 \pi \hbar}\right)^{3 / 2} \int d^{3} p\left(1+\frac{m c^{2}}{E}\right) e^{-\frac{|\vec{p}|^{2} d^{2}}{2 \hbar^{2}}+\frac{i}{\hbar} \vec{p} \cdot \vec{x}}\left(\begin{array}{c}
1 \\
0 \\
\frac{p_{z} c}{E+m c^{2}} \\
\frac{\left(p_{x}+i p_{y}\right) c}{E+m c^{2}}
\end{array}\right)
$$

We can approximate this state in the non-relativistic limit by computing these integrals assuming that $d \gg \frac{\hbar}{m c}$ so the momentum space Gaussian in the integrand suppresses modes where $|\vec{p}|^{2}$ is not $\ll m^{2} c^{2}{ }^{27}$

$$
\psi=\left(\frac{1}{\pi d^{2}}\right)^{3 / 4} e^{-|\vec{x}|^{2} / 2 d^{2}}\left(\begin{array}{c}
1 \\
0 \\
\frac{\hbar}{2 m c d^{2}} i z \\
\frac{\hbar}{2 m c d^{2}}(i x-y)
\end{array}\right)
$$

The total current density for this state (36), calculated via (24), is equal to the previous magnetization current density (31). Dividing this by the charge density for

\footnotetext{
${ }^{24}$ Although we have not discussed in detail the above-mentioned deeper modification of classical Dirac field theory needed to fully implement the idea of the Dirac sea (including holes), it is hard to see how that theory would allow for the electron's charge to be confined to a smaller volume. Holding fixed that the total charge in the Dirac field is $-e$, the presence of negative energy modes with positive charge density will only make it harder to localize the electron's charge in a small region.

${ }^{25}$ This Fourier decomposition is given in Bjorken \& Drell, 1964, section 3.3.

${ }^{26}$ As the negative frequency modes have simply been deleted, this new state is not normalized. In our classical terms, this means that the integral of the charge density over all space will not be $-e$. In the non-relativistic limit, the total charge will be close to $-e$.

${ }^{27}$ The same approximation can be arrived at by trusting only the first two components of (26) and using the positive frequency non-relativistic limit of the Dirac equation in Bjorken \& Drell (1964, eq. 1.31) to calculate the other two.
} 
(36) gives a charge velocity of

$$
\vec{v}_{d}^{q}=\frac{\frac{\hbar}{m d^{2}} \vec{x} \times \hat{z}}{1+\frac{\hbar^{2}}{m^{2} c^{2} d^{4}}|\vec{x}|^{2}} .
$$

This limits to (32) for $d \gg \frac{\hbar}{m c}$. Unlike (32), this is the actual charge velocity and not merely a contribution to it. The charge is really moving. The velocity in (37) is bounded and will not exceed the speed of light - as must be the case since the definition of the charge velocity (25) ensures that it cannot be superluminal. For $d \gg \frac{\hbar}{m c}$, the mass velocity derived from (36) using (22) will be as it was before (28). Thus, the charge rotates twice as fast as the mass.

This factor of two between mass and charge velocity is a general feature of states that describe electrons at rest in restricted Dirac field theory. What prevented us from reaching this conclusion in the previous section was that we had no reason to suppose the magnetization current density would be the dominant contribution to the total current density (29). The polarization current density could be significant as well. By restricting ourselves to superpositions of positive frequency modes, we have guaranteed that the polarization current density is small. To see why this is so, consider an arbitrary state of the Dirac field at an arbitrary time labeled $t=0, \psi(0)$. This state can be written as the sum of a superposition of positive frequency modes, $\psi_{+}$, and a superposition of negative frequency modes, $\psi_{-}$. In the non-relativistic limit, the time dependence of this state is given by

$$
\psi(t)=e^{\left(i m c^{2} / \hbar\right) t} \psi_{+}+e^{-\left(i m c^{2} / \hbar\right) t} \psi_{-} .
$$

The polarization current density for this state is

$$
\frac{i e \hbar}{2 m c} \frac{\partial}{\partial t}\left(\psi_{+}^{\dagger} \vec{\gamma} \psi_{+}+e^{-2\left(i m c^{2} / \hbar\right) t} \psi_{+}^{\dagger} \vec{\gamma} \psi_{-}+e^{2\left(i m c^{2} / \hbar\right) t} \psi_{-}^{\dagger} \vec{\gamma} \psi_{+}+\psi_{-}^{\dagger} \vec{\gamma} \psi_{-}\right) .
$$

If we forbid negative frequency modes, the cross terms are absent and the time derivative yields zero. Thus, in the non-relativistic limit the polarization current density is negligible.

In the previous section we were able to derive the factor of two between charge velocity and mass velocity only up to factors of $\gamma^{0}$. The reason these factors can be ignored is that $\gamma^{0}$ simply flips the sign of the third and fourth components of $\psi$ and these components - for a state composed of positive frequency modes in the non-relativistic limit - are much smaller than the first and second components.

At this point let us reflect on the role that the non-relativistic limit has played in the preceding analysis. This limit is not part of the general response to our first two obstacles. The fact that there is a minimum size for wave packets formed from positive frequency modes is not dependent on this limit, nor is the light-speed cap on charge velocity. The non-relativistic limit is, however, essential in explaining the electron's gyromagnetic ratio. The reason for this is that the gyromagnetic ratio we seek to account 
for only holds in the non-relativistic limit. ${ }^{28}$ Beyond this limit, the relationship between angular momentum and magnetic moment is more complex. In quantum mechanical terms, the relationship is given by the claim that the spin magnetic moment operator, $-\frac{e \hbar}{2 m c} \gamma^{0} \vec{\sigma}$, is $-\frac{e \gamma^{0}}{m c}$ times the spin angular momentum operator, $\frac{\hbar}{2} \vec{\sigma}$ (Ohanian, 1986, pg. 504; Frenkel, 1934, pg. 323). Expressed in terms of local expectation values, the local ratio of spin magnetic momentum to angular momentum is the ratio of $-\frac{e \hbar}{2 m c} \psi^{\dagger} \gamma^{0} \vec{\sigma} \psi$ to $\frac{\hbar}{2} \psi^{\dagger} \vec{\sigma} \psi$. In our classical field terminology, this is understood as the ratio of the spin magnetic moment density (30) to the spin angular momentum density (20).

\section{Conclusion}

The consensus about electron spin, which emerged long ago, is that the electron somehow acts like a spinning object without actually spinning. As Rojansky (1938, pg. 514) puts it in his textbook on quantum mechanics, after discussing spin angular momentum and spin magnetic moment in the context of the Dirac equation,

"In short, Dirac's equation automatically endows the electron with the properties that account for the phenomena previously ascribed to a hypothetical spinning motion of the electron." (original italics)

Here I have argued for a different interpretation. The Dirac equation does not somehow manage to account for these properties without positing a spinning electron. Instead, it explains just how the electron spins.

The obstacles to regarding the electron as spinning presented in the introduction were addressed as follows: Old estimates of the size of the electron made under the assumption that the electron's mass is primarily electromagnetic suggest that the electron would have to rotate superluminally in order to have the right angular momentum and magnetic moment. Actually, if the electron's mass is primarily electromagnetic we should focus on the rotation of the electromagnetic field's mass in calculating the electron's angular momentum and this mass cannot move superluminally. Also, the electron's mass is not primarily electromagnetic. When we move to better estimates of the electron's size - using the Dirac field to represent the state of the electron-we see that its minimum size is large enough that there is no need for superluminal rotation. Further, the definition of charge velocity for the Dirac field guarantees that the electron's charge will not move superluminally. The other obstacle was the fact that the electron's gyromagnetic ratio differs from the simplest classical estimate by a factor of two. On the account given here, this factor does not arise from some novel quantum revision to the basic physical principles defining angular momentum and magnetic moment, but is instead attributed to a false assumption in the simple classical estimate - the electron's mass and charge do not rotate at the same

\footnotetext{
${ }^{28}$ Standard explanations of the factor of two in the gyromagnetic ratio of the electron using the Dirac equation appeal to the non-relativistic limit (Bjorken \& Drell, 1964, section 1.4).
} 
rate.

Acknowledgments Thank you to Adam Becker, Dirk-André Deckert, John McGreevy, Lukas Nickel, Hans Ohanian, Laura Ruetsche, Roderich Tumulka, David Wallace, and anonymous referees for helpful feedback and discussion. This project was supported in part by funding from the President's Research Fellowships in the Humanities, University of California.

\section{References}

Barut, A.O. 1988. Quantum-electrodynamics based on self-energy. Physica Scripta, 1988(T21), 18-21.

Barut, A.O., \& Dowling, Jonathan P. 1990. Self-field quantum electrodynamics: The two-level atom. Physical Review A, 41(5), 2284-2294.

Bialynicki-Birula, Iwo. 1996. The photon wave function. Pages 313-322 of: Eberly, Joseph H., Mandel, Leonard, \& Wolf, Emil (eds), Coherence and Quantum Optics VII: Proceedings of the Seventh Rochester Conference on Coherence and Quantum Optics, held at the University of Rochester, June 7-10, 1995. Springer. An extended version of this article is available at http://arxiv.org/abs/quant-ph/0508202.

Bjorken, James D., \& Drell, Sydney D. 1964. Relativistic Quantum Mechanics. McGraw-Hill.

Bohm, David. 1953. Comments on an article of Takabayasi concerning the formulation of quantum mechanics with classical pictures. Progress of Theoretical Physics, 9(3), $273-287$.

Bohm, David, \& Hiley, Basil J. 1993. The Undivided Universe: An ontological interpretation of quantum theory. Routledge.

Carroll, Sean M. 2016. You should love (or at least respect) the Schrödinger equation. [Online; accessed 17-August-2017]

http://www.preposterousuniverse.com/blog/2016/08/15/

you-should-love-or-at-least-respect-the-schrodinger-equation/.

Colin, Samuel. 2004. Beables for quantum electrodynamics. Annales de la Fondation Louis de Broglie, 29(1-2), 273-296.

Corben, H.C. 1961. Spin in classical and quantum theory. Physical Review, 121(6), 1833-1839.

Crisp, Michael Dennis, \& Jaynes, E.T. 1969. Radiative effects in semiclassical theory. Physical Review, 179(5), 1253-1261. 
de Broglie, Louis. 1960. Non-Linear Wave Mechanics: A causal interpretation. Elsevier.

Dirac, Paul A.M. 1930. A theory of electrons and protons. Proceedings of the Royal Society of London A: Mathematical, Physical and Engineering Sciences, 126(801), $360-365$.

Dirac, Paul A.M. 1958. The Principles of Quantum Mechanics. 4 edn. Oxford University Press.

Duncan, Anthony. 2012. The Conceptual Framework of Quantum Field Theory. Oxford University Press.

Dürr, Detlef, Goldstein, Sheldon, Tumulka, Roderich, \& Zanghì, Nino. 2005. Bell-type quantum field theories. Journal of Physics A: Mathematical and General, 38(4), R1-R43.

Einstein, Albert. 1906. The principle of conservation of motion of the center of gravity and the inertia of energy. Annalen der Physik, 20, 627-633.

Feynman, Richard P., Leighton, Robert B., \& Sands, Matthew. 1964. The Feynman Lectures on Physics. Vol. II. Addison-Wesley Publishing Company.

Frenkel, J. 1934. Wave Mechanics: Advanced General Theory. Oxford University Press.

Giulini, Domenico. 2008. Electron spin or "classically non-describable two-valuedness". Studies In History and Philosophy of Science Part B: Studies In History and Philosophy of Modern Physics, 39(3), 557-578.

Good, Roland H., Jr. 1957. Particle aspect of the electromagnetic field equations. Physical Review, 105(6), 1914-1919.

Gottfried, Kurt, \& Weisskopf, Victor F. 1986. Concepts of Particle Physics. Vol. 2. Oxford University Press.

Goudsmit, Samuel A. 1998. The discovery of electron spin. Chap. 1, pages 2-12 of: Gareth R. Eaton, Sandra S. Eaton, Kev M. Salikhov (ed), Foundations Of Modern EPR. World Scientific.

Griffiths, David J. 1999. Introduction to Electrodynamics. 3 edn. Prentice Hall.

Griffiths, David J. 2005. Introduction to Quantum Mechanics. 2 edn. Pearson Prentice Hall.

Hatfield, Brian. 1992. Quantum Theory of Point Particles and Strings. Addison-Wesley. Frontiers in Physics, Volume 75.

Heitler, Walter H. 1954. The Quantum Theory of Radiation. 3 edn. Oxford University Press.

Holland, Peter R. 1993. The Quantum Theory of Motion: An account of the de Broglie-Bohm causal interpretation of quantum mechanics. Cambridge University 
Press.

Jackson, John D. 1999. Classical Electrodynamics. 3 edn. Wiley.

Jaynes, E.T. 1973. Survey of the present status of neoclassical radiation theory. Pages 35-81 of: Mandel, L., \& Wolf, E. (eds), Coherence and Quantum Optics. Plenum Press.

Lange, Marc. 2002. An Introduction to the Philosophy of Physics: Locality, Energy, Fields, and Mass. Blackwell.

MacGregor, Malcolm H. 1992. The Enigmatic Electron. Kluwer Academic Publishers.

Mignani, E, Recami, E, \& Baldo, M. 1974. About a Dirac-like equation for the photon according to Ettore Majorana. Lettere al Nuovo Cimento (1971-1985), 11(12), 568-572.

Ohanian, Hans C. 1986. What is spin? American Journal of Physics, 54(6), 500-505.

Pais, Abraham. 1989. George Uhlenbeck and the discovery of electron spin. Physics Today, 42(12), 34-40.

Pashby, Thomas. 2012. Dirac's prediction of the positron: a case study for the current realism debate. Perspectives on Science, 20(4), 440-475.

Pauli, Wolfgang. 1980. General Principles of Quantum Mechanics. Springer-Verlag.

Pearle, Philip. 1982. Classical electron models. Pages 211-295 of: Teplitz, Doris (ed), Electromagnetism: Paths to Research. Plenum Press.

Peskin, Michael E., \& Schroeder, Daniel V. 1995. An Introduction to Quantum Field Theory. Westview Press.

Poincaré, Henri. 1900. La théorie de Lorentz et le principe de réaction. Archives néerlandaises des sciences exactes et naturelles, 5, 252-278. Translation by S. Lawrence 2008 at http://www.physicsinsights.org/poincare-1900.pdf.

Rohrlich, Fritz. 2007. Classical Charged Particles. 3 edn. World Scientific.

Rojansky, Vladimir. 1938. Introductory Quantum Mechanics. Prentice-Hall.

Schweber, Silvan S. 1961. Introduction to Relativistic Quantum Field Theory. Harper \& Row.

Schweber, Silvan S. 1994. QED and the Men Who Made It: Dyson, Heynman, Schwinger, and Tomanaga. Princeton University Press.

Sebens, Charles T. forthcoming. Forces on fields. Studies in History and Philosophy of Modern Physics.

Struyve, Ward. 2010. Pilot-wave theory and quantum fields. Reports on Progress in Physics, 73(10), 106001. 
Teller, Paul. 1995. An interpretive introduction to quantum field theory. Princeton University Press.

Tomonaga, Sin-itiro. 1997. The Story of Spin. University of Chicago Press.

Tong, David. 2006. Lectures on Quantum Field Theory. http://www.damtp.cam.ac . uk/user/tong/qft.html [Online; accessed 2-August-2017].

Uhlenbeck, George E. 1976. Fifty years of spin: Personal reminiscences. Physics Today, $\mathbf{2 9}(6), 43-48$.

Valentini, Antony. 1992. On the pilot-wave theory of classical, quantum and subquantum physics. Ph.D. thesis, ISAS, Trieste, Italy.

Valentini, Antony. 1996. Pilot-wave theory of fields, gravitation, and cosmology. Pages 45-66 of: Cushing, James T., Fine, Arthur, \& Goldstein, Sheldon (eds), Bohmian Mechanics and Quantum Theory: An Appraisal. Kluwer Academic.

Wallace, David. forthcoming. The quantum theory of fields. In: Knox, Eleanor, \& Wilson, Alastair (eds), Handbook of Philosophy of Physics.

Weinberg, Steven. 1999. What is quantum field theory, and what did we think it was? Pages 241-251 of: Cao, Tian Yu (ed), Conceptual Foundations of Quantum Field Theory. Cambridge University Press.

Weisskopf, V. 1934a. Über die selbstenergie des elektrons. Zeitschrift für Physik, 89, $27-39$.

Weisskopf, V. 1934b. Berichtigung zu der arbeit: über die selbstenergie des elektrons. Zeitschrift für Physik, 90, 817-818.

Weisskopf, Victor F. 1939. On the self-energy and the electromagnetic field of the electron. Physical Review, 56(1), 72-85.

Wentzel, Gregor. 1949. Quantum Theory of Fields. Interscience Publishers.

Weyl, Hermann. 1932. The Theory of Groups and Quantum Mechanics. Dover. 